CRYSTALLOGRAPHIC COMMUNICATIONS

ISSN 2056-9890

Received 7 July 2020

Accepted 3 November 2020

Edited by H. Ishida, Okayama University, Japan

Keywords: crystal structure; benzoic acids; polymorphism; hydrogen bond; 2,6-dimethoxybenzoic acid.

CCDC reference: 2042162

Supporting information: this article has supporting information at journals.iucr.org/e

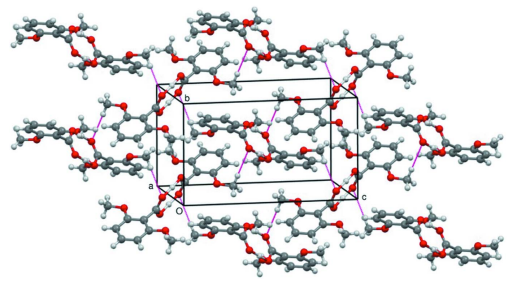

OPEN $\odot$ ACCESS

\section{Crystal structure and Hirshfeld surface analysis of a third polymorph of 2,6-dimethoxybenzoic acid}

\author{
Gustavo Portalone*
}

Chemistry Department, "Sapienza" University of Rome, P. le A. Moro 5, I-00185 Rome, Italy. *Correspondence e-mail: gustavo.portalone@uniroma1.it

A third crystalline form of the title compound, $\mathrm{C}_{9} \mathrm{H}_{10} \mathrm{O}_{4}$, crystallizing in the centrosymmetric monoclinic space group $P 2_{1} / c$, has been identified during screening for co-crystals. The asymmetric unit comprises a non-planar independent molecule with a synplanar conformation of the $\mathrm{OH}$ group. The sterically bulky $o$-methoxy substituents force the carboxy group to be twisted away from the plane of the benzene ring by $74.10(6)^{\circ}$. The carboxy group exhibits the acidic $\mathrm{H}$ atom disordered over two sites between two $\mathrm{O}$ atoms. A similar situation has been found for the second tetragonal polymorph reported [Portalone (2011). Acta Cryst. E67, o3394-03395], in which molecules with the $\mathrm{OH}$ group in a synplanar conformation form dimeric units via strong $\mathrm{O}-\mathrm{H} \cdots \mathrm{O}$ hydrogen bonds. In contrast, in the first orthorhombic form reported [Swaminathan et al. (1976). Acta Cryst. B32, 1897-1900; Bryan \& White (1982). Acta Cryst. B38, 1014-1016; Portalone (2009). Acta Cryst. E65, o327o328], the molecular components do not form conventional dimeric units, as an antiplanar conformation adopted by the $\mathrm{OH}$ group favors the association of molecules in chains stabilized by linear $\mathrm{O}-\mathrm{H} \cdots \mathrm{O}$ hydrogen bonds.

\section{Chemical context}

Until now, two polymorphs are known for 2,6-dimethoxybenzoic acid. Polymorph ( $\mathrm{I} \alpha)$ crystallizes in the orthorhombic space group $P 2_{1} 2_{1} 2_{1}$ with one molecule in the asymmetric unit (Swaminathan et al., 1976; Bryan \& White, 1982; Portalone, 2009). As a result of the antiplanar conformation adopted by the $\mathrm{OH}$ group, the molecular components are associated in the crystal in chains stabilized by linear $\mathrm{O}-\mathrm{H} \cdots \mathrm{O}$ hydrogen bonds. Polymorph $(\mathrm{I} \beta)$ crystallizes in the tetragonal space group $P 4_{1} 2{ }_{1} 2$ with one molecule in the asymmetric unit (Portalone, 2011). In the crystal of the second polymorph, the synplanar conformation of the $\mathrm{OH}$ group favours the formation of dimers through $\mathrm{O}-\mathrm{H} \cdots \mathrm{O}$ hydrogen bonds. In this article, it is reported the crystal structure of a third polymorph, $(\mathrm{I} \gamma)$, of 2,6-dimethoxybenzoic acid produced unexpectedly during an attempt to synthesize co-crystals of 5-fluorouracil with the title compound.<smiles>COc1cccc(OC)c1C(=O)O</smiles>

$\left(\mathrm{I}_{\alpha}\right)$ orthorhombic<smiles>COc1cccc(OC)c1C(=O)O</smiles>

$\left(I_{\beta}\right)$ tetragonal

$\left(I_{\gamma}\right)$ monoclinic 


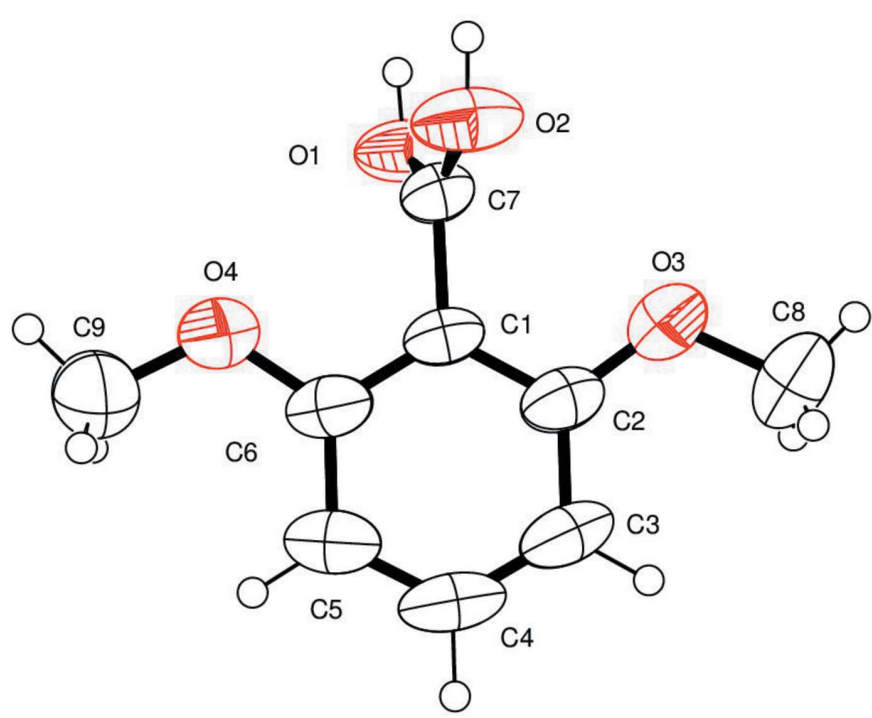

Figure 1

The molecular structure of $(\mathrm{I} \gamma)$, showing the atom-labeling scheme. Displacement ellipsoids are at the $50 \%$ probability level.

\section{Structural commentary}

The title compound $(\mathrm{I} \gamma)$ crystallizes in the monoclinic centrosymmetric space group $P 2_{1} / c$, and the asymmetric unit comprises a non-planar independent molecule. The carboxy group is twisted away from the plane of the benzene ring by $74.10(6)^{\circ}$ because of a significant steric hindrance of the two $o$-methoxy substituents (Fig. 1). The above angle between the planes is comparable with that found for the orthorhombic form, $56.12(9)^{\circ}$, and for the tetragonal form, $65.72(15)^{\circ}$. The
Table 1

Hydrogen-bond geometry $\left(\AA,^{\circ}\right)$.

\begin{tabular}{lllll}
\hline$D-\mathrm{H} \cdots A$ & $D-\mathrm{H}$ & $\mathrm{H} \cdots A$ & $D \cdots A$ & $D-\mathrm{H} \cdots A$ \\
\hline $\mathrm{O} 1-\mathrm{H} 1 \cdots \mathrm{O} 2^{\mathrm{i}}$ & $0.86(6)$ & $1.79(6)$ & $2.6411(15)$ & $174(4)$ \\
$\mathrm{O}^{\mathrm{i}}-\mathrm{H} 2 \cdots \mathrm{O} 1^{\mathrm{i}}$ & $0.81(6)$ & $1.84(6)$ & $2.6411(15)$ & $167(5)$ \\
$\mathrm{C} 9-\mathrm{H} 9 A \cdots \mathrm{O} 2^{\mathrm{ii}}$ & 0.97 & 2.60 & $3.571(3)$ & 178 \\
\hline
\end{tabular}

Symmetry codes: (i) $-x+1,-y+1,-z+1$; (ii) $-x+1, y-\frac{1}{2},-z+\frac{3}{2}$.

carboxy group, in which $\mathrm{OH}$ adopts a synplanar conformation similar to that observed for the tetragonal form, exhibits the carboxy $\mathrm{H}$ atom disordered over two sites between two $\mathrm{O}$ atoms. The pattern of bond lengths and bond angles of the phenyl ring is consistent with that reported in the structure determination of the two previously determined polymorphs, and a comparison of the present results with those obtained for similar benzene derivatives (Colapietro et al., 1984; Irrera et al., 2012; Portalone, 2012) shows no appreciable effects of the crystal environment on the ring deformation induced by substituents.

\section{Supramolecular features}

Analysis of the crystal packing of (I $\gamma$ ), (Fig. 2), shows that the molecular components form the conventional dimeric units observed in benzoic acids (Leiserowitz, 1976; Kanters et al., 1991; Moorthy et al., 2002). Indeed, the crystal structure is stabilized by strong intermolecular $\mathrm{O}-\mathrm{H} \cdots \mathrm{O}$ hydrogen bonds, which link inversion-related molecules into homodimers (Table 1). These homodimers are then joined by weak $\mathrm{C}-\mathrm{H} \cdots \mathrm{O}$ intermolecular interactions of graph-set motif

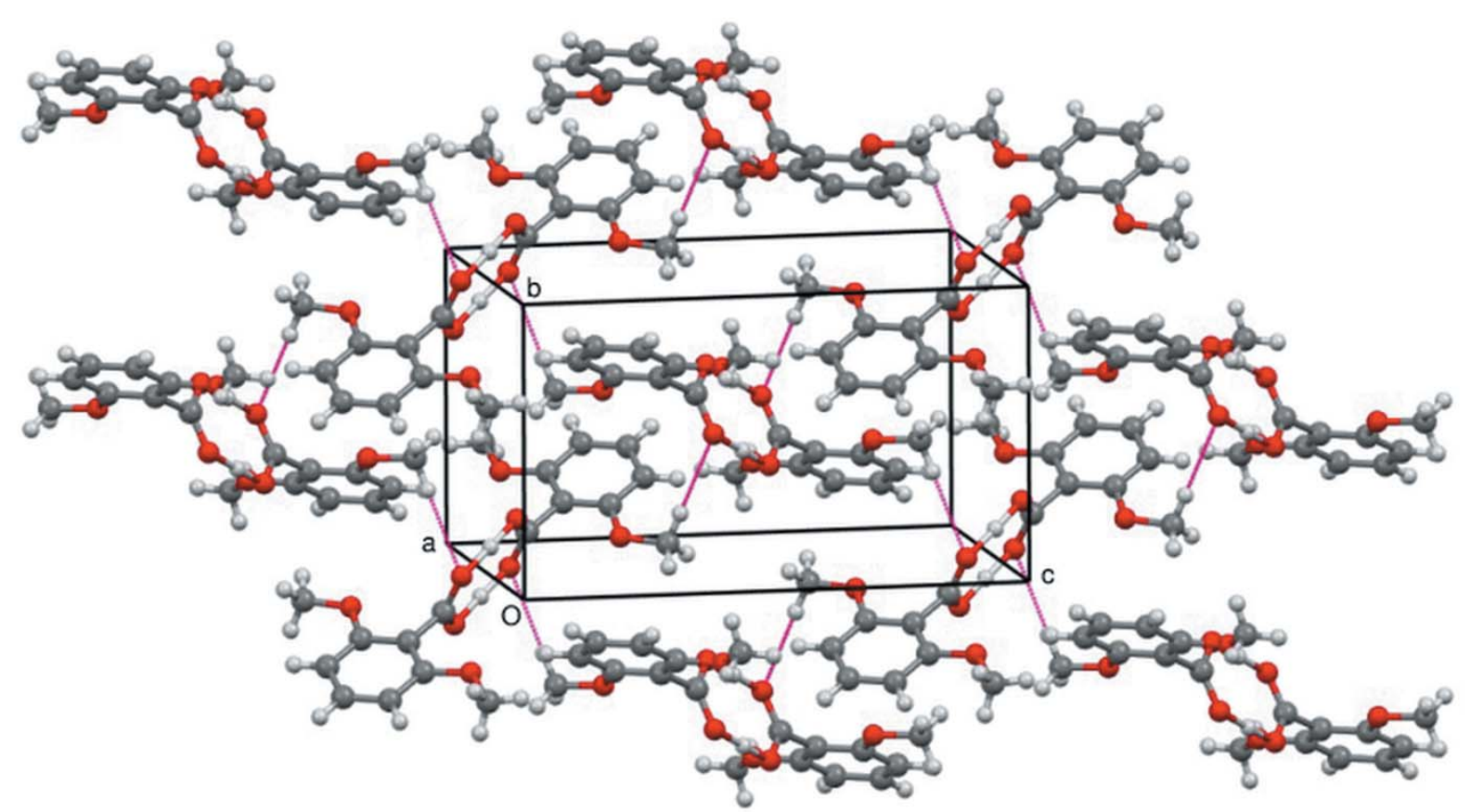

Figure 2

Crystal packing diagram for $(\mathrm{I} \gamma)$ viewed approximately down the $a$ axis. All atoms are shown as small spheres of arbitrary radii. Hydrogen bonding is indicated by red dashed lines. 
$R_{2}^{2}(6)$ between the methoxy and the carboxy groups of adjacent molecules to form a two-dimensional network parallel to the $b c$ plane.

The Hirshfeld surface analysis (Spackman \& Jayatilaka, 2009) was carried out using CrystalExplorer (Turner et al., 2017). The surface enables the visualization of intermolecular contacts over the surface by different colors and color intensity, and shorter and longer contacts are indicated as red and blue spots, respectively. In Fig. 3 are shown the 3D Hirshfeld surface, modeled by choosing one of the two equally disordered components and mapped over $d_{\text {norm, }}$ and the twodimensional fingerprint plots, which give the contribution of the interatomic contacts to the Hirshfeld surface. The most prominent interactions, due to strong $\mathrm{O}-\mathrm{H} \cdots \mathrm{O}$ hydrogen bonds, are shown by large and deep red spots on the surface. Small red spots on the surface indicate the areas where closecontact interactions due to weak $\mathrm{C}-\mathrm{H} \cdots \mathrm{O}$ hydrogen bonds take place. The $\mathrm{H} \cdots \mathrm{H}$ contacts, representing van der Waals interactions, and the $\mathrm{O} \cdots \mathrm{H} / \mathrm{H} \cdots \mathrm{O}$ contacts, representing intermolecular hydrogen bonds, are the most populated (a)
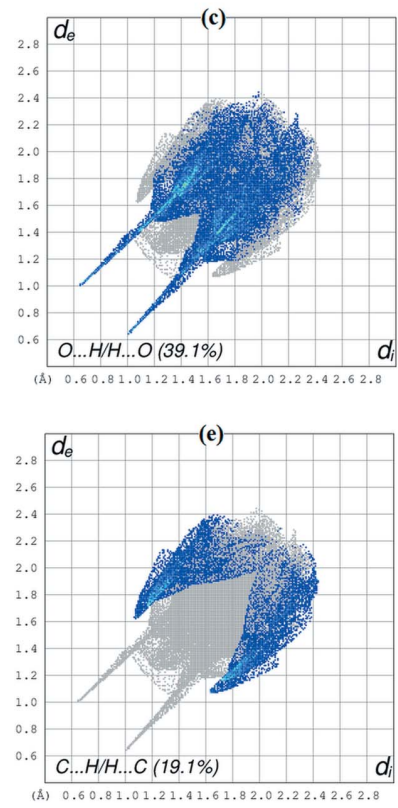

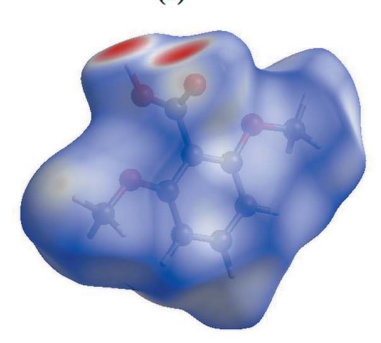

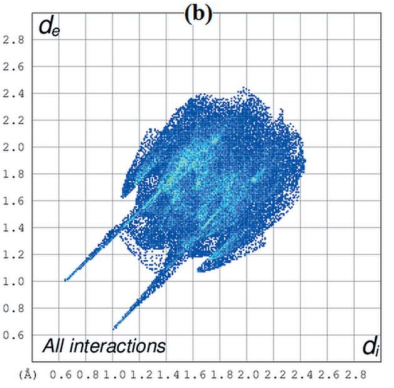
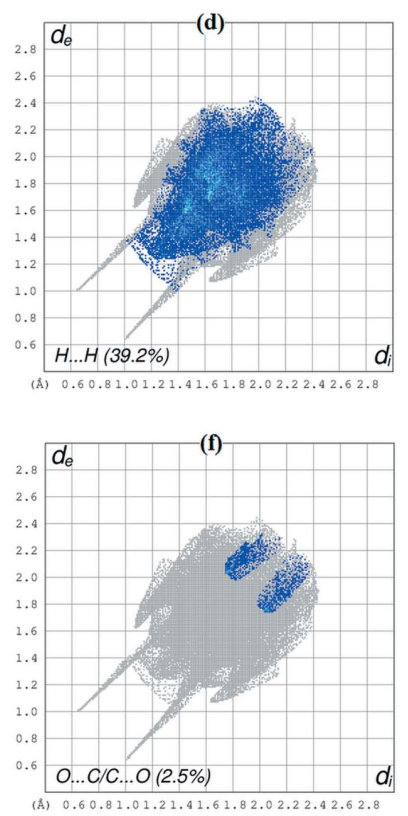

Figure 3

(a) A view of the three-dimensional Hirshfeld surface of the title compound mapped over $d_{\text {norm }}$ with a fixed color scale of -0.742 (red) to 1.283 (blue) a.u. $(b),(c),(d),(e)$ and $(f)$ : decomposed two-dimensional fingerprint plots for the title compound showing various close contacts and their proportional contributions.
Table 2

Experimental details.

Crystal data

Chemical formula

$M_{\mathrm{r}}$

Crystal system, space group

Temperature (K)

$a, b, c(\AA)$

$\beta\left({ }^{\circ}\right)$

$V\left(\AA^{3}\right)$

$Z$

Radiation type

$\mu\left(\mathrm{mm}^{-1}\right)$

Crystal size (mm)

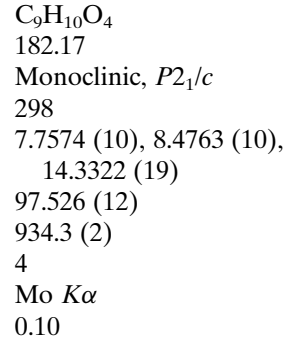

Data collection

Diffractometer

Absorption correction

$T_{\min }, T_{\max }$

No. of measured, independent and observed $[I>2 \sigma(I)]$ reflections

$R_{\text {int }}$

$(\sin \theta / \lambda)_{\max }\left(\AA^{-1}\right)$

Oxford Diffraction Xcalibur S
CCD
Multi-scan (CrysAlis RED; Rigaku
$\quad$ OD, 2018)
$0.970,0.999$
$9067,2708,1420$
0.040
0.704

$0.049,0.132,1.02$
2708
134
H atoms treated by a mixture of
independent and constrained
refinement
$0.14,-0.12$

$\Delta \rho_{\max }, \Delta \rho_{\min }\left(\mathrm{e} \AA^{-3}\right)$ $0.14,-0.12$

Computer programs: CrysAlis PRO (Rigaku OD, 2018), SIR2004 (Burla et al., 2005), SHELXL2014/7 (Sheldrick, 2015) and WinGX (Farrugia, 2012).

contacts and contribute 39.2 and $39.1 \%$ of the total intermolecular contacts, respectively. Other important contacts, such as $\mathrm{C} \cdots \mathrm{H} / \mathrm{H} \cdots \mathrm{C}(19.1 \%)$, also supplement the overall crystal packing. The contributions of the $\mathrm{O} \cdots \mathrm{C} / \mathrm{C} \cdots \mathrm{O}(2.5 \%)$ contacts are less significant.

\section{Database survey}

A search of crystal structure of 2,6-dimethoxy benzoic acid alone in the Cambridge Crystallographic Database (CSD version 5.41, May 2020 update; Groom et al., 2016) yielded four hits as crystalline polymorphs. Three were for the orthorhombic polymorph: DMOXBA (Swaminathan et al., 1976), DMOXBA01 (Bryan \& White, 1982) and DMOXBA02 (Portalone, 2009); the fourth one was for the tetragonal polymorph: DMOXBA03 (Portalone, 2011).

\section{Synthesis and crystallization}

Polymorph (I $\gamma)$ was formed from an unsuccessful co-crystallization between 2,6-dimethoxybenzoic acid and 5-fluorouracil. Colorless plate-like crystals were formed by the slow evaporation of an aqueous solution of 2,6-dimethoxybenzoic acid (1 mmol, Sigma Aldrich at 99\% purity) and 5-fluorouracil ( $1 \mathrm{mmol}$, Sigma Aldrich at $99 \%$ purity) in a 1:1 molar ratio. 


\section{Refinement}

Crystal data, data collection and structure refinement details are summarized in Table 2. All $\mathrm{H}$ atoms were identified in difference-Fourier maps, but in the refinement all $\mathrm{C}$-bound $\mathrm{H}$ atoms were placed in calculated positions, with $\mathrm{C}-\mathrm{H}=$ $0.97 \AA$, and refined as riding on their carrier atoms, with $U_{\text {iso }}(\mathrm{H})=1.2 U_{\text {eq }}\left(\mathrm{C}_{\text {phenyl }}\right)$ or $1.5 U_{\text {eq }}\left(\mathrm{C}_{\text {methyl }}\right)$. A rotating group model was applied to the methyl groups. The remaining two halves of the disordered $\mathrm{O}$-bound $\mathrm{H}$ atom, $\mathrm{H} 1$ and $\mathrm{H} 2$, were refined freely and their $U_{\text {iso }}$ values were kept equal to $1.2 U_{\text {eq }}(\mathrm{O})$. Site-occupation factors of $\mathrm{H} 1$ and $\mathrm{H} 2$ refined to 0.53 (3) and $0.47(3)$, respectively.

\section{References}

Bryan, R. F. \& White, D. H. (1982). Acta Cryst. B38, 1014-1016.

Burla, M. C., Caliandro, R., Camalli, M., Carrozzini, B., Cascarano, G. L., De Caro, L., Giacovazzo, C., Polidori, G. \& Spagna, R. (2005). J. Appl. Cryst. 38, 381-388.

Colapietro, M., Domenicano, A., Marciante, C. \& Portalone, G. (1984). Z. Naturforsch. Teil B, 39, 1361-1367.
Farrugia, L. J. (2012). J. Appl. Cryst. 45, 849-854.

Groom, C. R., Bruno, I. J., Lightfoot, M. P. \& Ward, S. C. (2016). Acta Cryst. B72, 171-179.

Irrera, S., Ortaggi, G. \& Portalone, G. (2012). Acta Cryst. C68, o447o451.

Kanters, J. A., Kroon, J., Hooft, R., Schouten, A., van Scijndel, J. A. M. \& Brandsen, J. (1991). Croat. Chem. Acta, 64, 353-370.

Leiserowitz, L. (1976). Acta Cryst. B32, 775-802.

Moorthy, J. N., Natarajan, R., Mal, P. \& Venugopalan, P. (2002). J. Am. Chem. Soc. 124, 6530-6531.

Portalone, G. (2009). Acta Cryst. E65, o327-o328.

Portalone, G. (2011). Acta Cryst. E67, o3394-03395.

Portalone, G. (2012). Acta Cryst. E68, o268-o269.

Rigaku OD (2018). CrysAlis PRO and CrysAlis RED. Rigaku Oxford Diffraction, Yarnton, England.

Sheldrick, G. M. (2015). Acta Cryst. C71, 3-8.

Spackman, M. A. \& Jayatilaka, D. (2009). CrystEngComm, 11, 1932.

Swaminathan, S., Vimala, T. M. \& Lotter, H. (1976). Acta Cryst. B32, 1897-1900.

Turner, M. J., McKinnon, J. J., Wolff, S. K., Grimwood, D. J., Spackman, P. R., Jayatilaka, D. \& Spackman, M. A. (2017). CrystalExplorer. University of Western Australia. http://hirshfeldsurface.net. 


\section{supporting information}

Acta Cryst. (2020). E76, 1823-1826 [https://doi.org/10.1107/S2056989020014553]

\section{Crystal structure and Hirshfeld surface analysis of a third polymorph of 2,6-di- methoxybenzoic acid}

\section{Gustavo Portalone}

\section{Computing details}

Data collection: CrysAlis PRO (Rigaku OD, 2018); cell refinement: CrysAlis PRO (Rigaku OD, 2018); data reduction: CrysAlis PRO (Rigaku OD, 2018); program(s) used to solve structure: SIR2004 (Burla et al., 2005); program(s) used to refine structure: SHELXL2014/7 (Sheldrick, 2015); molecular graphics: WinGX (Farrugia, 2012); software used to prepare material for publication: WinGX (Farrugia, 2012).

2,6-Dimethoxybenzic acid.

\section{Crystal data}

$\mathrm{C}_{9} \mathrm{H}_{10} \mathrm{O}_{4}$

$M_{r}=182.17$

Monoclinic, $P 2_{1} / c$

$a=7.7574(10) \AA$

$b=8.4763(10) \AA$

$c=14.3322(19) \AA$

$\beta=97.526(12)^{\circ}$

$V=934.3(2) \AA^{3}$

$Z=4$

\section{Data collection}

Oxford Diffraction Xcalibur S CCD diffractometer

Radiation source: Enhance (Mo) X-ray source

Graphite monochromator

Detector resolution: 16.0696 pixels $\mathrm{mm}^{-1}$

$\omega$ and $\varphi$ scans

Absorption correction: multi-scan (CrysAlis RED; Rigaku OD, 2018)

$T_{\min }=0.970, T_{\max }=0.999$

\section{Refinement}

Refinement on $F^{2}$

Least-squares matrix: full

$R\left[F^{2}>2 \sigma\left(F^{2}\right)\right]=0.049$

$w R\left(F^{2}\right)=0.132$

$S=1.02$

2708 reflections

134 parameters

0 restraints

Hydrogen site location: mixed
$F(000)=384$

$D_{\mathrm{x}}=1.295 \mathrm{Mg} \mathrm{m}^{-3}$

Mo $K \alpha$ radiation, $\lambda=0.710689 \AA$

Cell parameters from 1806 reflections

$\theta=2.9-32.6^{\circ}$

$\mu=0.10 \mathrm{~mm}^{-1}$

$T=298 \mathrm{~K}$

Tablets, colourless

$0.20 \times 0.14 \times 0.11 \mathrm{~mm}$

9067 measured reflections

2708 independent reflections

1420 reflections with $I>2 \sigma(I)$

$R_{\text {int }}=0.040$

$\theta_{\text {max }}=30.0^{\circ}, \theta_{\min }=2.9^{\circ}$

$h=-10 \rightarrow 10$

$k=-11 \rightarrow 6$

$l=-19 \rightarrow 20$

$\mathrm{H}$ atoms treated by a mixture of independent

and constrained refinement

$w=1 /\left[\sigma^{2}\left(F_{\mathrm{o}}^{2}\right)+(0.0439 P)^{2}+0.0492 P\right]$

where $P=\left(F_{\mathrm{o}}^{2}+2 F_{\mathrm{c}}^{2}\right) / 3$

$(\Delta / \sigma)_{\max }<0.001$

$\Delta \rho_{\max }=0.14 \mathrm{e} \AA^{-3}$

$\Delta \rho_{\min }=-0.12$ e $\AA^{-3}$ 
Extinction correction: SHELXL-2014/7

(Sheldrick 2015 $\backslash$ bbr000),

$\mathrm{Fc}^{*}=\mathrm{kFc}\left[1+0.001 \times \mathrm{Fc}^{2} \lambda^{3} / \sin (2 \theta)\right]^{-1 / 4}$

Extinction coefficient: $0.031(4)$

\section{Special details}

Geometry. All esds (except the esd in the dihedral angle between two 1.s. planes) are estimated using the full covariance matrix. The cell esds are taken into account individually in the estimation of esds in distances, angles and torsion angles; correlations between esds in cell parameters are only used when they are defined by crystal symmetry. An approximate (isotropic) treatment of cell esds is used for estimating esds involving l.s. planes.

Fractional atomic coordinates and isotropic or equivalent isotropic displacement parameters $\left(\AA^{2}\right)$

\begin{tabular}{llllll}
\hline & $x$ & $y$ & $z$ & $U_{\text {iso }} / U_{\text {eq }}$ & Occ. $(<1)$ \\
\hline O1 & $0.50254(16)$ & $0.32576(15)$ & $0.56267(9)$ & $0.0726(4)$ & \\
H1 & $0.446(6)$ & $0.372(5)$ & $0.515(3)$ & $0.087^{*}$ & $0.53(3)$ \\
O2 & $0.68711(16)$ & $0.52450(15)$ & $0.57631(9)$ & $0.0776(5)$ & \\
H2 & $0.643(7)$ & $0.571(6)$ & $0.530(4)$ & $0.093^{*}$ & $0.47(3)$ \\
O3 & $0.94633(16)$ & $0.24939(16)$ & $0.59823(10)$ & $0.0827(4)$ & \\
O4 & $0.50328(18)$ & $0.39851(17)$ & $0.76761(9)$ & $0.0900(5)$ & \\
C1 & $0.73003(19)$ & $0.31878(17)$ & $0.68819(11)$ & $0.0531(4)$ & \\
C2 & $0.8886(2)$ & $0.24603(19)$ & $0.68370(13)$ & $0.0622(5)$ & \\
C3 & $0.9743(2)$ & $0.1717(2)$ & $0.76309(15)$ & $0.0767(6)$ & \\
H3 & 1.0852 & 0.1199 & 0.7610 & $0.092^{*}$ & \\
C4 & $0.8991(3)$ & $0.1731(2)$ & $0.84415(15)$ & $0.0836(7)$ & \\
H4 & 0.9582 & 0.1201 & 0.8992 & $0.100^{*}$ & \\
C5 & $0.7430(3)$ & $0.2467(2)$ & $0.85095(13)$ & $0.0782(6)$ & \\
H5 & 0.6940 & 0.2474 & 0.9099 & $0.094^{*}$ & \\
C6 & $0.6574(2)$ & $0.3199(2)$ & $0.77134(12)$ & $0.0648(5)$ & \\
C7 & $0.63417(19)$ & $0.39559(18)$ & $0.60344(10)$ & $0.0508(4)$ & \\
C8 & $1.1123(3)$ & $0.1829(4)$ & $0.5897(2)$ & $0.1261(10)$ & \\
H8A & 1.2012 & 0.2385 & 0.6310 & $0.158(12)^{*}$ & \\
H8B & 1.1358 & 0.1925 & 0.5251 & $0.152(12)^{*}$ & \\
H8C & 1.1129 & 0.0723 & 0.6072 & $0.169(13)^{*}$ & \\
C9 & $0.4198(3)$ & $0.4098(3)$ & $0.84841(17)$ & $0.0986(7)$ & \\
H9A & 0.3944 & 0.3048 & 0.8698 & $0.139(10)^{*}$ & \\
H9B & 0.3122 & 0.4684 & 0.8337 & $0.129(9)^{*}$ & \\
H9C & 0.4950 & 0.4641 & 0.8976 & $0.155(12)^{*}$ & \\
& & & &
\end{tabular}

Atomic displacement parameters $\left(\AA^{2}\right)$

\begin{tabular}{lllllll}
\hline & $U^{11}$ & $U^{22}$ & $U^{33}$ & $U^{12}$ & $U^{13}$ & $U^{23}$ \\
\hline O1 & $0.0660(8)$ & $0.0702(8)$ & $0.0718(8)$ & $-0.0169(6)$ & $-0.0278(6)$ & $0.0167(6)$ \\
O2 & $0.0747(8)$ & $0.0651(8)$ & $0.0823(9)$ & $-0.0180(6)$ & $-0.0302(7)$ & $0.0257(7)$ \\
O3 & $0.0585(8)$ & $0.1008(10)$ & $0.0873(10)$ & $0.0180(7)$ & $0.0041(6)$ & $0.0170(8)$ \\
O4 & $0.0984(10)$ & $0.1059(11)$ & $0.0659(9)$ & $0.0408(8)$ & $0.0118(7)$ & $0.0165(7)$ \\
C1 & $0.0513(8)$ & $0.0503(8)$ & $0.0525(9)$ & $0.0003(7)$ & $-0.0128(7)$ & $0.0062(7)$ \\
C2 & $0.0512(9)$ & $0.0611(10)$ & $0.0693(12)$ & $-0.0009(7)$ & $-0.0108(8)$ & $0.0098(8)$ \\
C3 & $0.0579(10)$ & $0.0739(12)$ & $0.0904(14)$ & $0.0082(9)$ & $-0.0199(10)$ & $0.0192(10)$
\end{tabular}




\begin{tabular}{lllllll} 
C4 & $0.0835(14)$ & $0.0788(13)$ & $0.0775(14)$ & $0.0018(11)$ & $-0.0309(11)$ & $0.0234(10)$ \\
C5 & $0.0925(15)$ & $0.0788(12)$ & $0.0580(11)$ & $0.0040(11)$ & $-0.0103(9)$ & $0.0152(9)$ \\
C6 & $0.0712(11)$ & $0.0584(10)$ & $0.0599(11)$ & $0.0086(8)$ & $-0.0104(9)$ & $0.0068(8)$ \\
C7 & $0.0461(8)$ & $0.0509(8)$ & $0.0522(9)$ & $0.0012(7)$ & $-0.0057(6)$ & $0.0047(7)$ \\
C8 & $0.0704(16)$ & $0.173(3)$ & $0.138(3)$ & $0.0443(17)$ & $0.0250(17)$ & $0.037(2)$ \\
C9 & $0.1151(19)$ & $0.0953(17)$ & $0.0891(16)$ & $0.0249(15)$ & $0.0273(15)$ & $0.0135(14)$ \\
\hline
\end{tabular}

Geometric parameters $\left(\AA,{ }^{\circ}\right)$

\begin{tabular}{|c|c|c|c|}
\hline $\mathrm{O} 1-\mathrm{C} 7$ & $1.2563(17)$ & $\mathrm{C} 3-\mathrm{C} 4$ & $1.367(3)$ \\
\hline $\mathrm{O} 1-\mathrm{H} 1$ & $0.86(6)$ & $\mathrm{C} 3-\mathrm{H} 3$ & 0.9700 \\
\hline $\mathrm{O} 2-\mathrm{C} 7$ & $1.2472(18)$ & $\mathrm{C} 4-\mathrm{C} 5$ & $1.377(3)$ \\
\hline $\mathrm{O} 2-\mathrm{H} 2$ & $0.81(6)$ & $\mathrm{C} 4-\mathrm{H} 4$ & 0.9700 \\
\hline $\mathrm{O} 3-\mathrm{C} 2$ & $1.359(2)$ & $\mathrm{C} 5-\mathrm{C} 6$ & $1.389(2)$ \\
\hline $\mathrm{O} 3-\mathrm{C} 8$ & $1.425(2)$ & $\mathrm{C} 5-\mathrm{H} 5$ & 0.9700 \\
\hline $\mathrm{O} 4-\mathrm{C} 6$ & $1.363(2)$ & $\mathrm{C} 8-\mathrm{H} 8 \mathrm{~A}$ & 0.9701 \\
\hline $\mathrm{O} 4-\mathrm{C} 9$ & $1.402(2)$ & $\mathrm{C} 8-\mathrm{H} 8 \mathrm{~B}$ & 0.9701 \\
\hline $\mathrm{C} 1-\mathrm{C} 6$ & $1.383(2)$ & $\mathrm{C} 8-\mathrm{H} 8 \mathrm{C}$ & 0.9701 \\
\hline $\mathrm{C} 1-\mathrm{C} 2$ & $1.385(2)$ & $\mathrm{C} 9-\mathrm{H} 9 \mathrm{~A}$ & 0.9701 \\
\hline $\mathrm{C} 1-\mathrm{C} 7$ & $1.4885(19)$ & C9-H9B & 0.9701 \\
\hline $\mathrm{C} 2-\mathrm{C} 3$ & $1.391(2)$ & $\mathrm{C} 9-\mathrm{H} 9 \mathrm{C}$ & 0.9701 \\
\hline $\mathrm{C} 7-\mathrm{O} 1-\mathrm{H} 1$ & $117(3)$ & $\mathrm{O} 4-\mathrm{C} 6-\mathrm{C} 1$ & $115.07(14)$ \\
\hline $\mathrm{C} 7-\mathrm{O} 2-\mathrm{H} 2$ & $124(3)$ & $\mathrm{O} 4-\mathrm{C} 6-\mathrm{C} 5$ & $124.95(19)$ \\
\hline $\mathrm{C} 2-\mathrm{O} 3-\mathrm{C} 8$ & $118.50(17)$ & $\mathrm{C} 1-\mathrm{C} 6-\mathrm{C} 5$ & $119.97(17)$ \\
\hline $\mathrm{C} 6-\mathrm{O} 4-\mathrm{C} 9$ & $119.91(16)$ & $\mathrm{O} 2-\mathrm{C} 7-\mathrm{O} 1$ & $123.27(13)$ \\
\hline $\mathrm{C} 6-\mathrm{C} 1-\mathrm{C} 2$ & $120.51(14)$ & $\mathrm{O} 2-\mathrm{C} 7-\mathrm{C} 1$ & $119.20(13)$ \\
\hline $\mathrm{C} 6-\mathrm{C} 1-\mathrm{C} 7$ & $118.92(14)$ & $\mathrm{O} 1-\mathrm{C} 7-\mathrm{C} 1$ & $117.53(14)$ \\
\hline $\mathrm{C} 2-\mathrm{C} 1-\mathrm{C} 7$ & $120.57(16)$ & $\mathrm{O} 3-\mathrm{C} 8-\mathrm{H} 8 \mathrm{~A}$ & 109.5 \\
\hline $\mathrm{O} 3-\mathrm{C} 2-\mathrm{C} 1$ & $115.62(14)$ & $\mathrm{O} 3-\mathrm{C} 8-\mathrm{H} 8 \mathrm{~B}$ & 109.5 \\
\hline $\mathrm{O} 3-\mathrm{C} 2-\mathrm{C} 3$ & $124.61(17)$ & $\mathrm{H} 8 \mathrm{~A}-\mathrm{C} 8-\mathrm{H} 8 \mathrm{~B}$ & 109.5 \\
\hline $\mathrm{C} 1-\mathrm{C} 2-\mathrm{C} 3$ & $119.74(18)$ & $\mathrm{O} 3-\mathrm{C} 8-\mathrm{H} 8 \mathrm{C}$ & 109.5 \\
\hline $\mathrm{C} 4-\mathrm{C} 3-\mathrm{C} 2$ & $118.64(18)$ & $\mathrm{H} 8 \mathrm{~A}-\mathrm{C} 8-\mathrm{H} 8 \mathrm{C}$ & 109.5 \\
\hline $\mathrm{C} 4-\mathrm{C} 3-\mathrm{H} 3$ & 120.7 & $\mathrm{H} 8 \mathrm{~B}-\mathrm{C} 8-\mathrm{H} 8 \mathrm{C}$ & 109.5 \\
\hline $\mathrm{C} 2-\mathrm{C} 3-\mathrm{H} 3$ & 120.7 & $\mathrm{O} 4-\mathrm{C} 9-\mathrm{H} 9 \mathrm{~A}$ & 109.5 \\
\hline $\mathrm{C} 3-\mathrm{C} 4-\mathrm{C} 5$ & $122.81(16)$ & $\mathrm{O} 4-\mathrm{C} 9-\mathrm{H} 9 \mathrm{~B}$ & 109.5 \\
\hline $\mathrm{C} 3-\mathrm{C} 4-\mathrm{H} 4$ & 118.6 & $\mathrm{H} 9 \mathrm{~A}-\mathrm{C} 9-\mathrm{H} 9 \mathrm{~B}$ & 109.5 \\
\hline $\mathrm{C} 5-\mathrm{C} 4-\mathrm{H} 4$ & 118.6 & $\mathrm{O} 4-\mathrm{C} 9-\mathrm{H} 9 \mathrm{C}$ & 109.5 \\
\hline $\mathrm{C} 4-\mathrm{C} 5-\mathrm{C} 6$ & $118.33(19)$ & $\mathrm{H} 9 \mathrm{~A}-\mathrm{C} 9-\mathrm{H} 9 \mathrm{C}$ & 109.5 \\
\hline $\mathrm{C} 4-\mathrm{C} 5-\mathrm{H} 5$ & 120.8 & $\mathrm{H} 9 \mathrm{~B}-\mathrm{C} 9-\mathrm{H} 9 \mathrm{C}$ & 109.5 \\
\hline $\mathrm{C} 6-\mathrm{C} 5-\mathrm{H} 5$ & 120.8 & & \\
\hline $\mathrm{C} 8-\mathrm{O} 3-\mathrm{C} 2-\mathrm{C} 1$ & $177.2(2)$ & $\mathrm{C} 9-\mathrm{O} 4-\mathrm{C} 6-\mathrm{C} 5$ & $0.1(3)$ \\
\hline $\mathrm{C} 8-\mathrm{O} 3-\mathrm{C} 2-\mathrm{C} 3$ & $-4.9(3)$ & $\mathrm{C} 2-\mathrm{C} 1-\mathrm{C} 6-\mathrm{O} 4$ & $178.43(14)$ \\
\hline $\mathrm{C} 6-\mathrm{C} 1-\mathrm{C} 2-\mathrm{O} 3$ & $178.84(15)$ & $\mathrm{C} 7-\mathrm{C} 1-\mathrm{C} 6-\mathrm{O} 4$ & $-2.2(2)$ \\
\hline $\mathrm{C} 7-\mathrm{C} 1-\mathrm{C} 2-\mathrm{O} 3$ & $-0.5(2)$ & $\mathrm{C} 2-\mathrm{C} 1-\mathrm{C} 6-\mathrm{C} 5$ & $-0.5(3)$ \\
\hline $\mathrm{C} 6-\mathrm{C} 1-\mathrm{C} 2-\mathrm{C} 3$ & $0.9(2)$ & $\mathrm{C} 7-\mathrm{C} 1-\mathrm{C} 6-\mathrm{C} 5$ & $178.92(15)$ \\
\hline $\mathrm{C} 7-\mathrm{C} 1-\mathrm{C} 2-\mathrm{C} 3$ & $-178.51(14)$ & $\mathrm{C} 4-\mathrm{C} 5-\mathrm{C} 6-\mathrm{O} 4$ & $-179.32(17)$ \\
\hline
\end{tabular}


supporting information

$\begin{array}{ll}\mathrm{O} 3-\mathrm{C} 2-\mathrm{C} 3-\mathrm{C} 4 & -178.01(17) \\ \mathrm{C} 1-\mathrm{C} 2-\mathrm{C} 3-\mathrm{C} 4 & -0.2(3) \\ \mathrm{C} 2-\mathrm{C} 3-\mathrm{C} 4-\mathrm{C} 5 & -0.8(3) \\ \mathrm{C} 3-\mathrm{C} 4-\mathrm{C} 5-\mathrm{C} 6 & 1.2(3) \\ \mathrm{C} 9-\mathrm{O} 4-\mathrm{C} 6-\mathrm{C} 1 & -178.74(18)\end{array}$

$\mathrm{C} 4-\mathrm{C} 5-\mathrm{C} 6-\mathrm{C} 1$
$\mathrm{C} 6-\mathrm{C} 1-\mathrm{C} 7-\mathrm{O} 2$
$\mathrm{C} 2-\mathrm{C} 1-\mathrm{C} 7-\mathrm{O} 2$
$\mathrm{C} 6-\mathrm{C} 1-\mathrm{C} 7-\mathrm{O} 1$
$\mathrm{C} 2-\mathrm{C} 1-\mathrm{C} 7-\mathrm{O} 1$

$-0.5(3)$

$106.30(19)$

$-74.3(2)$

$-73.7(2)$

$105.66(18)$

Hydrogen-bond geometry $\left(A,{ }^{\circ}\right)$

\begin{tabular}{lllll}
\hline$D-\mathrm{H} \cdots A$ & $D-\mathrm{H}$ & $\mathrm{H} \cdots A$ & $D \cdots A$ & $D-\mathrm{H}^{\cdots} A$ \\
\hline $\mathrm{O} 1-\mathrm{H} 1 \cdots \mathrm{O} 2^{\mathrm{i}}$ & $0.86(6)$ & $1.79(6)$ & $2.6411(15)$ & $174(4)$ \\
$\mathrm{O} 2-\mathrm{H} 2 \cdots \mathrm{O} 1^{\mathrm{i}}$ & $0.81(6)$ & $1.84(6)$ & $2.6411(15)$ & $167(5)$ \\
$\mathrm{C} 9-\mathrm{H} 9 A \cdots \mathrm{O} 2^{\mathrm{ii}}$ & 0.97 & 2.60 & $3.571(3)$ & 178 \\
\hline
\end{tabular}

Symmetry codes: (i) $-x+1,-y+1,-z+1$; (ii) $-x+1, y-1 / 2,-z+3 / 2$. 